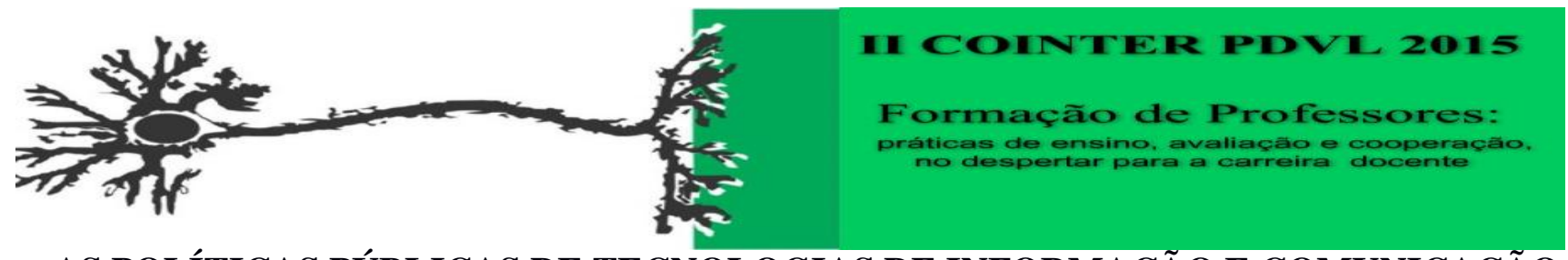

\title{
AS POLÍTICAS PÚBLICAS DE TECNOLOGIAS DE INFORMAÇÃO E COMUNICAÇÃO NA EDUCAÇÃO BRASILEIRA: ENTRAVES E PERSPECTIVAS
}

\author{
Apresentação: Comunicação Oral \\ Luiz Alberto da Silva Junior .email: junior mineiros@hotmail.com
}

\begin{abstract}
Resumo
Este ensaio busca fazer uma revisão sobre parte das políticas públicas que discutem a inserção das tecnologias de informação e comunicação (TIC) na educação brasileira, partindo do pressuposto emergente acerca da falta de relação entre políticas públicas para o uso das TIC e formação de professores, conforme diversos pesquisadores tem encontrado em seus trabalhos. Em primeiro lugar, discute-se a tecnologia do ponto de vista social e político, levantando pontos como a ideologia e o uso da escola como espaço de perpetuação de culturas dominantes, tendo como aporte as categorias da sociologia da educação propostas por Bourdieu. Com base nisso, estuda-se as bases legais, especificamente a LDB e as DCNEM, no que se refere às habilidades e competências inerentes ao uso das tecnologias, buscando encontrar pontos fortes e fracos nessa legislação. Além disso, apresenta-se algumas perspectivas atuais sobre e-governo e políticas públicas. Por fim, dirige-se o foco para as políticas específicas de distribuição de computadores portáteis ou tablets nas redes públicas municipais e estaduais, discutindo as principais dificuldades para a utilização pedagógica dessas tecnologias e traçando perspectivas para novos estudos tendo como base a experiência relatada na literatura internacional. Corroboram com os argumentos apresentados os dados oriundos do Comitê Gestor da Internet, cujo mapeamento permite traçar tendências sobre o uso da internet e das tecnologias no contexto educacional brasileiro.
\end{abstract}

Palavras-Chave: Tecnologias de informação e comunicação, políticas públicas, formação de professores.

\section{Sociedade e tecnologia}

Pierre Bourdieu, importante sociólogo francês do século XX, descreveu que mudanças na sociedade produzem mudanças dentro do espaço escolar. Por muito tempo, se pensou que a escola fosse um ambiente neutro dentro da comunidade, da mesma forma que se entendia a aprendizagem como mero fruto do esforço individual de cada estudante. Bourdieu, então, provoca inquietações ao apresentar sua perspectiva de que a escola age como sistema de reprodução das desigualdades sociais (BOURDIEU; PASSERON, 2013).

Nesse sentido, a escola passa a ser um campo estratégico dentro da sociedade pois se comporta como um agente transformador e motor do desenvolvimento da nação, conforme também aponta Tornaghi (2008). Ao assumir esse papel, a escola se torna o principal interlocutor da cultura tida como dominante. Em outras palavras, uma das funções dessa escola é levar a cultura da classe dominante para as massas da sociedade. 
Por conta disso, as políticas públicas educacionais são elaboradas visando atingir certos objetivos que são definidos pelos atores dominantes nesse processo e que, devido ao avanço tecnológico do último século, tem despendido esforços em inserir as tecnologias de informação e comunicação nas redes de ensino e nos seus diversos níveis. É importante ressaltar que existem sentidos implícitos nessas iniciativas. Concordamos, assim, com Cunha e Miranda quando pontuam que:

O desenvolvimento econômico e social, pelo aumento do emprego e da renda e da melhoria da qualidade de vida em todos os extratos da sociedade, não pode prescindir da integração de uma política pública que considere as TIC como instrumento essencial para a sua promoção. Entretanto, tem claro os autores deste trabalho que, como regra geral, estes movimentos não nascem de demandas internas da sociedade brasileira; antes, surgem aqui e ali em nosso país obedecendo a uma agenda internacional e se desenvolvem, numa ou noutra área, de acordo com os interesses dos diversos atores da sociedade. (CUNHA; MIRANDA, 2013, p. 558)

Os mesmos autores apontam para a configuração governamental de uma sociedade de informação do Brasil (Ibid., p. 545) através de uma série de medidas, das quais: projetos de infraestrutura, estabelecimento de padrões nacionais e adequação aos padrões internacionais, políticas tecnológicas, legislações específicas e medidas de implantação ou hesitação de recursos tecnológicos. No campo educacional - foco deste trabalho - as principais medidas dessa configuração tem sido a inserção das tecnologias de informação e comunicação nas escolas e universidades. Há informações de políticas de distribuição de notebooks e tablets para professores e/ou alunos em pelo menos cinco estados através dos portais na internet das respectivas secretarias estaduais de educação: Minas Gerais, Pernambuco, Rio de Janeiro, São Paulo e Sergipe.

Do ponto de vista educacional, a literatura já aponta para a necessária discussão acerca da utilização pedagógica dessas tecnologias de informação e comunicação. Leão (2011) chama a atenção para o fato de que o computador, a internet, o tablet, não passam de ferramentas. O simples enxerto dessas ferramentas na sala de aula não produz novos aprendizados. Pelo contrário, é necessário que o professor possua saberes específicos para construir as estratégias didáticas pertinentes, visando assim a efetiva construção do conhecimento. Em outras palavras, não é o tablet que faz o aluno aprender mais ou melhor, é a estratégia de uso que o professor adota.

Leão (2011) mostra, ainda, que um dos primeiros pontos que professores e diretores alegam para o pouco uso dos recursos disponíveis nas escolas é que os alunos não têm maturidade ou não possuem conhecimentos procedimentais para utilizá-los, assim opta-se por manter o material livre do risco de ser "danificado". Essa problemática da capacitação é, sem dúvida, uma barreira para a utilização pedagógica e que, ao que parece, não tem um peso nas decisões públicas. Cunha e 
Miranda argumentam na mesma direção ao afirmar que:

Os esforços governamentais para o uso de internet (e de todos os meios digitais) podem ser inócuos se não for considerado o enorme desafio que representa o grande contingente da população brasileira que está alijado de um acesso aos meios de comunicação, a um computador, e mesmo às habilidades de sua utilização e até às de leitura e escrita que se fazem necessárias para o uso. (CUNHA; MIRANDA, 2013, p. 553)

Além disso, existe uma série de outras dificuldades para a efetiva implantação das tecnologias na educação básica. Azevedo, Bernardino Junior e Daróz (2014) apresentam, do ponto de vista da análise do discurso, que há conflitos entre os discursos governamentais e do professorado. Segundo os autores, as políticas públicas condicionam o discurso do professor, levando-o à propagação da ideia de que as tecnologias são importantes e contribuem para o aprendizado. No entanto, durante a prática diária, o professor sofre um processo de desidentificação quando percebe que a falta de infra-estrutura, a falta de formação, a falta de apoio da gestão escolar, dentre outros aspectos, dificultam e até impedem a utilização das tecnologias na sua sala de aula. Melo (2012, p. 95) caminha na mesma direção quando aponta que "no âmbito do trabalho a tarefa é determinada ou prescrita pelo outro - por exemplo, o Ministério da Educação -, e não pelo docente; por isso, há sempre uma distância entre o trabalho prescrito e o trabalho realizado pelo professor".

Nesse viés, justifica-se a crescente demanda por pesquisas que pretendem avaliar esse tipo de política pública, identificar possíveis fragilidades e apontar novas perspectivas. Assim, desenvolvemos este trabalho guiado pela seguinte questão de investigação: como se estabelece a discussão na literatura acerca das políticas públicas para a inclusão das tecnologias de informação e comunicação no ensino?

\section{As políticas públicas de tecnologias de informação e comunicação}

As políticas públicas voltadas para as tecnologias sofreram um processo de disseminação e massificação no Brasil ainda no final do século XX. Embora se acredite que elas representem um avanço recente, as primeiras políticas brasileiras voltadas para o uso das mesmas datam dos anos 70 ainda no regime militar (MORAES, 1999). Como exemplos pioneiros, podemos tomar a Universidade Aberta do Brasil e a TV Escola, as quais foram iniciativas públicas ainda no século passado. Contudo, é no limiar do século XXI que emergem políticas, investigações e debates sobre o tema.

Alguns pesquisadores (LAIA; CUNHA; NOGUEIRA; MAZZON, 2011; CUNHA; MIRANDA, 2013) tem utilizado o termo e-governo para se referir às políticas governamentais específicas para as tecnologias de informação e comunicação. Em resumo, são políticas voltadas 
para a inclusão digital, a melhoria da qualidade de vida e das relações sociais através da tecnologia. No campo educacional, o e-governo tem se configurado através do incentivo ao uso de recursos tecnológicos tanto por parte de professores (como incentivos à infra-estrutura física das escolas) quanto por parte de alunos (através da distribuição de mídias e hardware).

No entanto, os avanços das tecnologias e das exigências do mercado não acompanharam o desenvolvimento dessas políticas, culminando nas problemáticas já apresentadas. Sobre isso, o Comitê Gestor da Internet no Brasil conclui que:

Pesquisas universitárias, resultados de experiências internacionais, novas pressões do mercado, mas, sobretudo, a evolução vertiginosa das tecnologias acelerou ou atropelou os seus usos que dificultaram em muito o desenvolvimento de uma política de Estado, coerente e unificada. Aliem-se a tais dificuldades e facilidades o crescimento enorme da rede pública de atendimento aos alunos e as dificuldades de acesso às redes web, e mudança contínua no mercado dos computadores de mesa até os tablets nestes últimos 20 anos (ALMEIDA; FRANCO, 2014, p. 48).

Dessa forma, é perceptível um movimento assíncrono entre avanço tecnológico e avanço das políticas públicas. Para entender como essas políticas avançaram, precisamos levantar as principais bases legais e o que elas pregam acerca das tecnologias de informação e comunicação. A partir disso, é possível entender como as diversas instâncias governamentais estão se apropriando ou não dessa legislação.

A principal base legal para a educação brasileira, a Lei de Diretrizes e Bases da Educação Nacional (LDB), Lei 9394/96, na seção 3, do Ensino Fundamental, aponta como um de seus objetivos: a compreensão do ambiente natural e social, do sistema político, da tecnologia, das artes e dos valores em que se fundamenta a sociedade (BRASIL, 1996, p. 12, grifo nosso). Essa definição ainda é arcaica, uma vez que apenas pontua o termo "tecnologia" sem uma discussão aprofundada de como seria essa compreensão. Além disso, existe a confusão comum entre técnica e tecnologia, apontada nos trabalhos de Ataíde e Mesquita (2013).

Outra base legal que fundamenta as práticas e políticas educacionais são as Diretrizes Curriculares Nacionais para o Ensino Médio (DCNEM). Em seu artigo 10 fica estabelecido que algumas das habilidades e competências do educando devem ser:

Entender os princípios das tecnologias da comunicação e da informação, associálas aos conhecimentos científicos, às linguagens que lhes dão suporte e aos problemas que se propõem solucionar; Entender a natureza das tecnologias da informação como integração de diferentes meios de comunicação, linguagens e códigos, bem como a função integradora que elas exercem na sua relação com as demais tecnologias; Entender o impacto das tecnologias da comunicação e da informação na sua vida, nos processos de produção, no desenvolvimento do conhecimento e na vida social; Aplicar as tecnologias da comunicação e da informação na escola, no trabalho e em outros contextos relevantes para sua vida. (CNE, 1998, p. 4, grifo nosso) 
A proposta das DCNEM se aproxima bastante do objetivo que a literatura tem atribuído às tecnologias de informação e comunicação. Em primeiro lugar, é preciso que o estudante entenda as tecnologias como ferramentas criadas pelo homem em um processo dialético de transformação do mundo e de si mesmo (COLL, 2012; BRAGA, 2013). Além disso, é preciso utilizá-las associadas aos conhecimentos científicos para que possam contribuir na resolução de problemas, contribuindo assim para a formação do cidadão crítico que deve estar apto a aplicar o saber escolar nos diversos problemas reais do seu cotidiano (SANTOS; MORTIMER, 2000). Por fim, faz parte da competência tecnológica o entendimento de que a tecnologia é uma criação humana e, portanto, perpassam ideologias, concepções de mundo e interesses sócio-políticos, negando a premissa de que o avanço tecnológico sempre busca a melhoria da qualidade da vida através de uma ciência neutra e desprovida de interesses (MORAES, 1999; SANTOS; MORTIMER, 2000).

Diante disso tudo podemos nos indagar: como os professores deverão trabalhar para que os estudantes desenvolvam tais competências? Ao apresentar essa questão, entramos em outro entrave para a efetivação das políticas públicas de tecnologias na educação: a formação dos professores. Ao que parece, essas políticas públicas se debruçam mais no financiamento dos equipamentos do que no investimento na formação continuada dos professores. Sobre isso, Almeida e Franco alertam que:

Mesmo que houvesse total financiamento para aquisição de equipamentos e para formação de professores, ele não seria suficiente para que o uso crie um todo orgânico curricular. Muitas diretrizes políticas para a implantação das TIC supõem, equivocadamente, que o aparelhamento dos espaços escolares vai induzir espontaneamente que professores e alunos as usem de forma articulada ao currículo e adequadas aos fins da aprendizagem que se falou até aqui. É engano (ALMEIDA; FRANCO, 2013).

Isso evidencia que o simples investimento financeiro não garante a plena utilização futura das tecnologias. Pelo contrário, como já foi dito, a falta de capacitação dos estudantes e dos professores pode provocar o abandono dos materiais comprados. No levantamento feito pelo Comitê Gestor da Internet no Brasil foi constatado que a "formação específica para o uso das TIC foi feita por apenas $52 \%$ dos professores, e que $73 \%$ deles pagaram a própria formação. Apenas $22 \%$ da formação feita pelos docentes foi oferecida pelo governo ou pelas Secretarias de Educação (ALMEIDA; FRANCO, 2013, p. 44). Essas porcentagens corroboram para a hipótese de que o investimento em formação continuada tem sido escasso e pode ser um dos motivos pelos quais as tecnologias não estão sendo utilizadas de maneira satisfatória.

Seguindo na contramão dessa problemática, a nova tendência das esferas governamentais 
tem sido o pesado investimento financeiro na aquisição e distribuição de computadores portáteis ou tablets para os estudantes das redes públicas municipais e estaduais em detrimento da capacitação dos profissionais da educação para o uso desses recursos. Não se pode negar a eficácia que tais tecnologias provocam no ensino e na aprendizagem. Karsenti $(2014$, p. 68) ao investigar a política de distribuição de computadores portáteis para estudantes da educação básica de Québec conseguiu identificar dez benefícios: 1) Motivação dos alunos; 2) Acesso a recursos de informação e de educação para estudantes e professores; 3) Habilidades de escrita dos alunos; 4) Métodos de trabalho eficazes dos alunos; 5) Sensação de competência dos alunos; 6) Aprendizagem individualizada; 7) Qualidade do trabalho dos alunos; 8) Maior facilidade de comunicação, cooperação e trabalho em equipe para professores e alunos; 9) Melhor preparação dos jovens para o futuro e para o seu próprio futuro; 10) Criatividade dos alunos.

Contudo, é preciso que tais políticas sejam avaliadas tanto pelos governos quanto pelos pesquisadores educacionais a fim de se estabelecer metas e perspectivas, bem como entender as limitações e fragilidades para cada caso específico. Na literatura internacional a discussão sobre a inserção de computadores portáteis e tablets já está bem estabelecida. Têm-se investigações sobre o uso de tablets por crianças na pré-escola (COUSE; CHEN, 2010; BESCHORNER; HUTCHISON, 2013), percepções de professores em formação (AKSU, 2014; ÇUHADAR, 2014), uso de tablets em estudantes com necessidades especiais (JOHNSON, 2013), avaliação da política pública do governo da Turquia de distribuir tablets aos estudantes (PAMUK et al, 2013), uso de tablets no ensino de matemática (GALLIGAN, McDONALD, TAYLOR, 2010), no ensino de línguas (SAVAS, 2014) e no ensino de artes e design (SOULELES et al, 2015), além de percepções de estudantes quanto ao uso de tablets no ensino superior (MANG; WARDLEY, 2013).

No Brasil essa discussão ainda é incipiente, o que se evidencia pelo baixo número de artigos científicos publicados sobre o tema, bem como através da pouca problematização encontrada nos trabalhos já dispostos na literatura. Em geral, são trabalhos pontuais que apresentam alguma estratégia para o uso de determinada tecnologia. Nesse sentido, se classificam como utilitaristas segundo alguns pesquisadores. Dessa forma, pode-se perceber que o estabelecimento efetivo das políticas públicas ainda não se deu por completo no contexto brasileiro.

\section{Palavras finais}

Diante da revisão de literatura exposta, é possível traçar alguns aspectos sobre as tecnologias de informação e comunicação na educação a partir de pressupostos teóricos e empíricos. No que diz respeito à natureza das políticas públicas e da própria tecnologia enquanto artefatos humanos, é importante destacar seu caráter político e ideológico. Assim, não se pode dissociar a política dos 
interesses implícitos e dos objetivos inconscientes presentes nesses investimentos. Identificá-los é o primeiro passo para trabalhar a efetividade de tais políticas.

O segundo ponto importante a se observar em futuras análises é o caráter pedagógico das políticas, isto é, como elas contribuem para a aprendizagem dos estudantes. Para isso, é preciso trabalhar em professores e estudantes as habilidades técnicas necessárias para o manuseio das tecnologias, a desconstrução de crenças sobre a tecnologia como salvação da educação e, sobretudo, investir em formação continuada. Além disso, é preciso minimizar a diferença entre o discurso governamental e o discurso professoral através de políticas que sejam possíveis, de fato, de serem implementadas.

Por fim, é preciso aprofundar as investigações acadêmicas sobre o impacto dessas tecnologias e dessas políticas no aprendizado dos estudantes e na prática dos professores. Tomando como exemplo a produção internacional que já se debruça sobre aspectos diversos do uso de recursos tecnológicos, é preciso que os pesquisadores nacionais auxiliem os governos fazendo a avaliação pedagógica dessas intervenções, propondo caminhos e perspectivas para as próximas décadas.

\section{Referências}

AKSU, H. H. An evaluation into the views of candidate mathematics teachers over "tablet computers" to be applied in secondary schools. The Turkish Online Journal of Educational Technology, v. 13, n. 1, 2014.

ALMEIDA, F. J.; FRANCO, M. G. Tecnologias para a educação e políticas curriculares de estado. In: BARBOSA, A. F (coord). Pesquisa sobre o uso das tecnologias de informação e comunicação nas escolas brasileiras: TIC Educação 2013. 1. ed. São Paulo: Comitê Gestor da Internet no Brasil, 2014.

ATAÍDE, J. F.; MESQUITA, N. A. S. O laboratório de Informática: conflitos circunstanciais na visão docente sob a perspectiva de Bourdieu. In: Encontro Nacional de Pesquisa em Educação em Ciências, IX, 2013, Águas de Lindóia-SP. Anais.

AZEVEDO, N. P. G.; BERNARDINO JÚNIOR, F. M.; DARÓZ, E. P. O professor e as novas tecnologias na perspectiva da análise do discurso: (des)encontros em sala de aula. Revista Linguagem em Discurso, v. 14, n. 1, 2014.

BESCHORNER, B.; HUTCHISON, A. iPads as a literacy teaching tool in early childhood. International Journal of Education in Mathematics, Science and Technology, v. 1, n. 1, 2013

BOURDiEU, P.; PASSERON, J. C. A reprodução: Elementos para uma teoria do sistema de ensino. $6^{\text {a }}$ ed. Petrópolis: Editora Vozes, 2013.

BRAGA, D. B. Ambientes digitais: reflexões téoricas e práticas. $1^{\text {a }}$ ed. São Paulo: Editora Cortez, 
2013.

BRASIL. Lei n. 9394, de 20 de dezembro de 1996. Estabelece as diretrizes e bases da educação nacional. Presidência da República. Brasília, Fernando Henrique Cardoso.

COLL, C.; MONEREO, C. Psicologia da educação virtual: aprender e ensinar com as tecnologias da informação e comunicação. Porto Alegre: Artmed, 2012.

CONSELHO NACIONAL DE EDUCAÇÃO. Resolução CEB n. 3, de 26 de junho de 1998. Institui as Diretrizes Curriculares Nacionais para o Ensino Médio. Câmara de Educação Básica. Brasília, Ulysses de Oliveira Panisset.

COUSE, L. J.; CHEN, D. W. A Tablet Computer for young children? Exploring its viability for early childhood education. Journal of Research on Technology in Education, v. 43, n. 1, 2010.

ÇUHADAR, C. Information Technologies Pre-service Teachers' acceptance of Tablet PCs as an innovative learning tool. Journal Educational Sciences: Theory\&Practice, v. 14, n. 2, 2014.

CUNHA, M. A. V. C.; MIRANDA, P. R. M. O uso de TIC pelos governos: uma proposta de agenda de pesquisa a partir da produção acadêmica e da prática nacional. Revista Organizações\&Sociedade, v. 20, n. 66, 2013.

GALLIGAN, L.; LOCH, B.; McDONALD, C.; TAYLOR, J. A. The use of tablet and related technologies in mathematics teaching. Australian Senior Mathematics Journal, v. 24, n. 1, 2010.

JOHNSON, G. M. Using Tablet Computers with elementary school students with special needs: The practices and perceptions of special education teachers and teacher assistants. Canadian Journal of Learning and Technology, v. 39, n. 4, 2013.

KARSENTI, T. Os benefícios educacionais e os desafios dos projetos de distribuição de computadores portáteis individuais nos ensinos primário e secundário. In: BARBOSA, A. F (coord). Pesquisa sobre o uso das tecnologias de informação e comunicação nas escolas brasileiras: TIC Educação 2013. 1. ed. São Paulo: Comitê Gestor da Internet no Brasil, 2014.

LAIA, M. M.; CUNHA, M. A. V. C.; NOGUEIRA, A. R. R.; MAZZON, J. A. Electronic government policies in Brazil: Context, ICT Management and outcomes. Revista de Administração de Empresas, v. 51, n. 1, 2011.

LEÃO, M. B. C (org). Tecnologias na educação: uma abordagem crítica para uma atuação prática. Recife: Editora Universitária da UFRPE, 2011.

MANG, C. F.; WARDLEY, L. J. Student perceptions of using Tablet technology in post-secondary classes. Canadian Journal of Learning and Technology, v. 39, n. 4, 2013.

MELO, G. C. V. O uso das TIC no trabalho de professores universitários de língua inglesa. Revista Brasileira de Linguística Aplicada, v. 12, n. 1, 2012.

MORAES, R. A. A política de informática na educação brasileira: do nacionalismo ao neoliberalismo. Revista Linhas Críticas, v. 5, n. 9, 1999. 
PAMUK, S.; ÇAKIR, R.; ERGUN, M.; YILMAZ, H. B. AYAS, C. The use of Tablet PC and interactive board from the perspectives of teachers and students: Evaluation of the FATIH Project. Educational Sciences: Theory \& Practice, v. 13, n. 3, 2013.

SANTOS, W. L.; MORTIMER, E. F. Uma análise de pressupostos teóricos da abordagem CTS no contexto da educação brasileira. Revista Ensaio, v. 2, n. 2, 2000.

SAVAS, P. Tablet PCs as intructional tools in english as a foreign language education. The Turkish Online Journal of Educational Technology, v. 13, n. 1, 2014.

SOULELES, N.; SAVVA, S.; WATTERS, H.; ANNESLEY, A.; BULL, B. A phenomenographic investigation on the use of iPads among undergraduate art and design students. British Journal of Educational Technology, v. 46, n. 1, 2015.

TORNAGHI, A. Proposta Pedagógica. In: MINISTÉRIO DA EDUCAÇÃO. Salto para o futuro: Educação digital e tecnologias da informação e da comunicação. Ano XVIII, boletim 18, 2008. 